\title{
Impact of oxide and electrode materials on the switching characteristics of oxide ReRAM devices
}

\author{
Elia Ambrosi, (iD Alessandro Bricalli, (D) Mario Laudato (iD) \\ and Daniele lelmini (iD *
}

Received 28th May 2018, Accepted 23rd July 2018

DOI: $10.1039 / \mathrm{c} 8 \mathrm{fd} 00106 \mathrm{e}$

\begin{abstract}
Resistive switching random-access memory (ReRAM) is one of the most promising technologies for non-volatile memories. Thanks to the low power and high speed operation, the high density CMOS-compatible integration, and the high cycling endurance, the ReRAM technology is becoming a strong candidate for high-density storage arrays and novel in-memory computing systems. However, ReRAM suffers from cycle-to-cycle switching variability and noise-induced resistance fluctuations, leading to insufficient read margin between the programmed resistive states. To overcome the existing challenges, a deep understanding of the roles of the ReRAM materials in the device characteristics is essential. To better understand the role of the switching layer material in controlling ReRAM performance and reliability, this work compares $\mathrm{SiO}_{x^{-}}$and $\mathrm{HfO}_{2}$-based ReRAM at fixed geometry and electrode materials. $\mathrm{Ti} / \mathrm{HfO}_{2} / \mathrm{C}$ and $\mathrm{Ti} / \mathrm{SiO}_{x} / \mathrm{C}$ devices are compared from the point of view of the forming process, switching characteristics, resistance variability, and temperature stability of the programmed states. The results show clear similarities for the two different oxides, including a similar resistance window and stability at high temperatures, thus suggesting a common nature of the switching mechanism, highlighting the importance of the electrodes. On the other hand, the oxide materials play a clear role in the forming, breakdown, and variability characteristics. The discrimination between the role of the oxide and the electrode materials in the ReRAM allows ReRAM optimization via materials engineering to be better explored for future memory and computing applications.
\end{abstract}

\section{Introduction}

In recent years, resistive switching memory (ReRAM) devices have emerged as strong candidates for next generation high-density storage technologies. ${ }^{1-3}$ The resistance switching mechanism enables not only high-density non-volatile 
memory with high speed, high endurance, and low power, but can also be exploited for implementing novel computing paradigms such as in-memory computing and neuromorphic computing. ${ }^{4-6}$ Currently, ReRAM technologies are facing significant challenges due to switching variability and resistance fluctuations, which require major breakthroughs at the levels of materials engineering and device physics. ${ }^{7-9}$

In previous works, we proposed a silicon oxide-based ReRAM, in which an ultra-thin layer of $\mathrm{SiO}_{x}$ is sandwiched between an inert carbon bottom electrode (BE) and an active titanium top electrode (TE). ${ }^{10}$ Electrical switching between a high-resistance state (HRS) and a low-resistance state (LRS) was attributed to the oxidation of titanium in the TE and migration of Ti ions into the solid electrolyte, where they react to form a Ti-rich conductive filament (CF). This type of switching is equivalent to the well-known conductive bridge RAM (CBRAM), or electrical metallization cell (EMC). The adoption of a TE made of $\mathrm{Cu}$ and $\mathrm{Ag}$ was shown to lead to volatile switching, which might be applicable for selector devices in high density crosspoint arrays ${ }^{11}$ and synaptic devices. ${ }^{12}$

In the static regime, $\mathrm{SiO}_{x}$-based ReRAM shows an outstanding resistance window exceeding $10^{4}$. On the other hand, a ratio of about 10 is typically found in $\mathrm{HfO}_{2}$-based ReRAM, where switching is generally assumed to be due to the oxidation/reduction of metallic elements, known as the valence change memory (VCM) mechanism. ${ }^{13}$ The larger resistance window of the $\mathrm{SiO}_{x}$ ReRAM is in agreement with previous studies which attribute a larger window to CBRAM-type devices. ${ }^{14}$ A high resistance window is essential in ReRAM to allow for sufficient read margin between the HRS and the LRS, ensuring a sufficient immunity from post-programming fluctuations of resistance. ${ }^{9} \mathrm{SiO}_{x}$ ReRAM has also shown a relatively low variability, an excellent cycling endurance, and an outstanding stability at elevated temperature, ${ }^{10}$ compared with $\mathrm{HfO}_{2}$ ReRAM. ${ }^{15,16}$ To enable a high device reliability and a deeper understanding of the materials parameters controlling the resistance window, ReRAM materials should be compared at fixed geometry and top/bottom electrode types.

In this work, we compare $\mathrm{SiO}_{x}$-based ReRAM and $\mathrm{HfO}_{2}$-based ReRAM on the same test vehicle, which is a one-transistor/one-resistor (1T1R) structure which enables a tight control of the programming current, namely the compliance current $I_{\mathrm{C}}$. A comprehensive comparison is conducted, covering the forming operation, the switching characteristics, and the retention behavior at elevated temperature. We also investigate cycle-to-cycle variability by means of the relative standard deviation, namely the ratio between the standard deviation $\sigma_{\mathrm{R}}$ and the resistance $R$, which is the standard metric for stochastic variation of programmed states in ReRAM. Our results indicate that the $\mathrm{SiO}_{x}$ and $\mathrm{HfO}_{2}$ ReRAM show remarkably similar device properties when compared on the same test vehicle. Similar performance includes the large resistance window and the good retention at elevated temperature. This performance similarity suggests that the ReRAM behavior is mostly dictated by the Ti TE and its migration controlling the filamentary switching in the two devices. On the other hand, the forming, breakdown and variability characteristics show remarkable dependence on the oxide material, which enables discrimination between the role of the oxide and of the electrode materials in the ReRAM switching mechanism. 


\section{Device structures}

Fig. 1 illustrates the two ReRAM structures studied in this work, namely the $\mathrm{SiO}_{x}$-based ReRAM (a) and the $\mathrm{HfO}_{2}$-based ReRAM (b). Both devices were deposited on pre-patterned substrates with metal-oxide semiconductor (MOS) field-effect transistors (FETs) to enable the fabrication of 1T1R structures. After a $300{ }^{\circ} \mathrm{C}$ vacuum pre-processing of the substrates, a thin dielectric film was deposited by electron-beam evaporation from silicon monoxide $\left(\mathrm{SiO}_{x}, x \approx 1\right)$ or hafnium dioxide $\left(\mathrm{HfO}_{2}\right)$ solid targets on top of a graphitic carbon bottom electrode (BE) of area $70 \times 70 \mathrm{~nm}^{2} .^{\mathbf{1 0}}$ The Ti TE was deposited on top of the oxide layer without breaking the vacuum from the previous oxide deposition. The Ti TE had a thickness of $50 \mathrm{~nm}$. A tungsten plug connects the BE to the drain of the FET to enable an integrated $1 \mathrm{~T} 1 \mathrm{R}$ structure. The $\mathrm{HfO}_{2}$ film was deposited with a thickness of $t_{\mathrm{ox}}=5 \mathrm{~nm}$ or $t_{\mathrm{ox}}=10 \mathrm{~nm}$, whereas the $\mathrm{SiO}_{x}$ film thickness was $t_{\mathrm{ox}}=5 \mathrm{~nm}$.

After the fabrication process, the devices were electrically characterized in a probe station by means of a Keysight B1500A semiconductor parameter analyzer.

\section{Forming and switching characteristics}

\subsection{Forming}

Fig. 2(a) shows the $I-V$ curves measured on the pristine cell for 30 devices for $t_{\mathrm{ox}}=5 \mathrm{~nm}$. The voltage was applied to the TE and increased at a rate of about $1 \mathrm{~V} \mathrm{~s}^{-1}$. The gate of the FET in the 1T1R structure was biased with a voltage $V_{\mathrm{G}}$ in the range between $1 \mathrm{~V}$ and $1.6 \mathrm{~V}$, corresponding to a compliance current $I_{\mathrm{C}}$ between 1 and $70 \mu \mathrm{A}$, while the source was grounded.

Initially, the pristine device shows very low leakage current, limited by the instrument resolution for a TE voltage below $1.5 \mathrm{~V}$. The current increases for increasing positive voltage, as a result of Poole-Frenkel conduction at localized states. Forming appears as a sudden step in the $I-V$ curves, at a characteristic voltage $V_{\text {FORM }}$, triggering a soft breakdown which brings the device in the LRS.

(a)

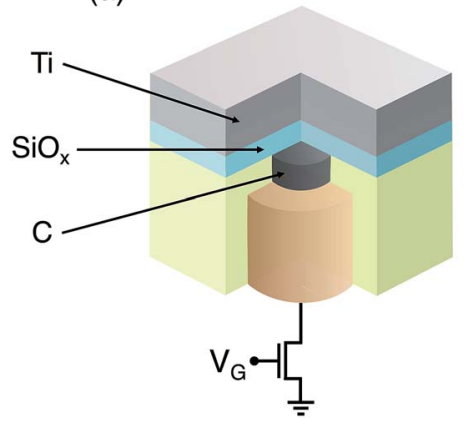

(b)

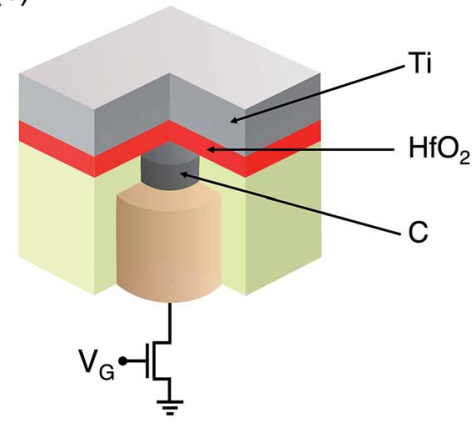

Fig. 1 Schematic illustration of the (a) $\mathrm{SiO}_{x}$-based, and (b) $\mathrm{HfO}_{2}$-based ReRAM devices. The dielectric switching layer is deposited on top of a graphitic $\mathrm{C}$ bottom electrode. The Ti top electrode serves as the active electrode supplying Ti impurity defects for the filament formation. 
(a)

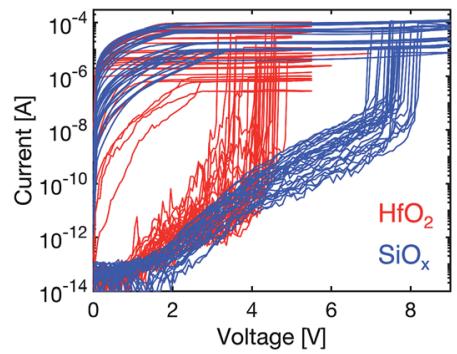

(b)

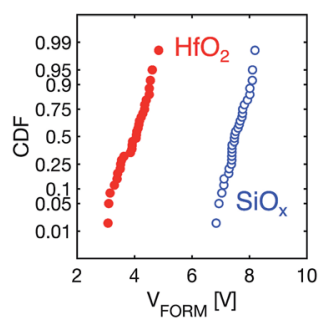

(c)

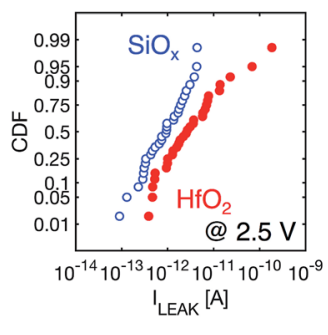

Fig. 2 (a) $I-V$ characteristics of the forming process in the $\mathrm{HfO}_{2}$ and $\mathrm{SiO}_{x}$ stacks of thickness $t_{\mathrm{ox}}=5 \mathrm{~nm}$. (b) Experimental cumulative probability of the forming voltages $V_{\text {FORM }}$ in different devices. (c) Experimental cumulative probability of the leakage current $I_{\text {LEAK }}$ extracted at fixed voltage $V=2.5 \mathrm{~V}$ from the $I-V$ curves, in different devices. The two stacks show comparable leakage currents, slightly higher in the $\mathrm{HfO}_{2}$ device, while the forming voltage is higher in $\mathrm{SiO}_{x}$.

The forming operation marks the initial formation of a CF, the size of which is controlled by the maximum current $I_{\mathrm{C}}$ through the device, which also prevents the irreversible breakdown of the ReRAM device upon forming.

From the $I-V$ curves in Fig. 2(a), one can notice a large difference in $V_{\text {FORM, }}$, which is about $\approx 4 \mathrm{~V}$ for $\mathrm{HfO}_{2}$ and $\approx 7.5 \mathrm{~V}$ for $\mathrm{SiO}_{x}$. Fig. 2(b) shows the cumulative distributions of $V_{\text {FORM }}$ for the ReRAM devices, which also reveal a higher distribution spread for $\mathrm{HfO}_{2}$ compared to $\mathrm{SiO}_{x}$. On the other hand, the leakage current $I_{\text {LEAK }}$ before forming is comparable in the two dielectric materials, as confirmed by the probability distributions in Fig. 2(c) showing $I_{\text {LEAK }}$ at $V=2.5 \mathrm{~V}$. The leakage current shows a larger distribution spread and higher noise in $\mathrm{HfO}_{2}$, possibly related to the higher instability of defects such as oxygen vacancies compared to $\mathrm{SiO}_{x}$. The lower $V_{\mathrm{FORM}}$ in $\mathrm{HfO}_{2}$ results in a lower forming current, which is about $1 \mathrm{nA}$ in $\mathrm{HfO}_{2}$ compared to 10 to $100 \mathrm{nA}$ in $\mathrm{SiO}_{x}$. Such a result may be due to a smaller energy barrier for defect migration in $\mathrm{HfO}_{2}$, compared to $\mathrm{SiO}_{x}$, which is also supported by the higher current noise and the lower breakdown voltage (see Sec. 4.1).

\subsection{Switching characteristics}

Fig. 3 shows our interpretation of the switching mechanism in a filamentary bipolar ReRAM. The forming operation results in the formation of a continuous defect-rich conductive filament (CF) of diameter $\phi$ connecting the two electrodes. This corresponds to the low resistance state (LRS) of the memory device depicted in Fig. 3(a). During the reset operation, the CF is disrupted thus resulting in a transition to the high resistance state (HRS) in Fig. 3(b). During the reset transition, initiated by the application of a negative voltage to the TE, positively-ionized defects (Ti impurities and/or oxygen vacancies) migrate towards the TE in response to the applied field, thus leaving behind a highly-resistive depleted gap of length $\Delta \cdot{ }^{17}$ The application of a positive voltage to the TE induces the migration of defects from the TE into the depleted gap, leading to the reconnection of the CF.

Fig. 4 shows the measured $I-V$ curves for 50 cycles for $\mathrm{HfO}_{2}$ with $t_{\mathrm{ox}}=10 \mathrm{~nm}$ (a) and $\mathrm{SiO}_{x}$ with $t_{\mathrm{ox}}=5 \mathrm{~nm}$ (b). After the forming process the ReRAM device can be 
(a)

(b)

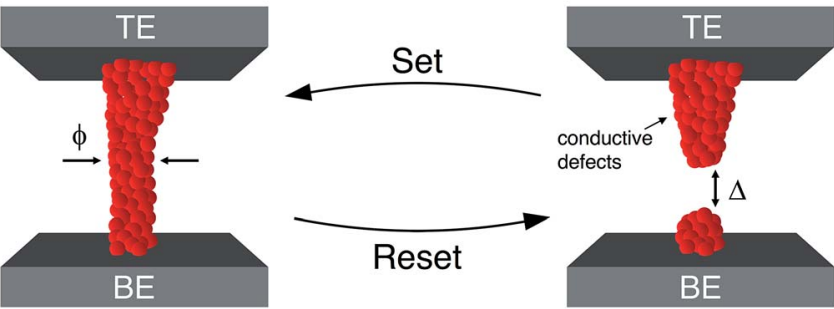

Fig. 3 Schematic representation of the set/reset operations in the ReRAM device. (a) In the low resistance state (LRS) a continuous conductive filament (CF) connects the top electrode (TE) and the bottom electrode (BE). The reset operation causes the disconnection of the CF and the consequent transition to the high resistance state (HRS) (b). The set operation brings the device back to the LRS.

brought back to the HRS by the application of a negative voltage to the TE, inducing a reset transition. When the negative voltage exceeds $V_{\text {reset }}$ a gradual decrease of current takes place, due to the field-induced migration and consequent disruption of the CF. The HRS resistance is about $10^{8} \Omega$ at the read voltage $V_{\text {read }}=-0.5 \mathrm{~V}$. The application of a positive voltage induces the set process in the ReRAM leading to the LRS at the characteristic set voltage $V_{\text {set }}$. The abrupt nature of the set transition can be explained by a positive feedback effect, where the electric field induces defect migration toward the depleted gap, which in turn causes an increase of the electric field and temperature by Joule heating. This

(a)

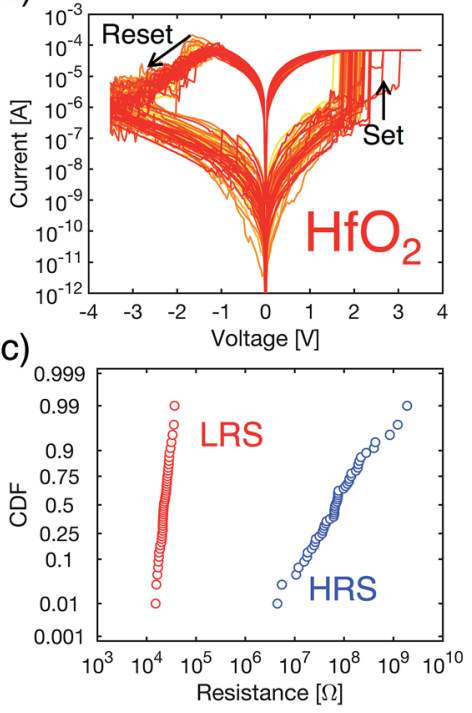

(b)

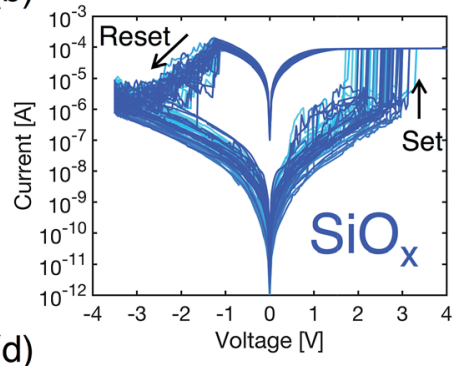

(d)

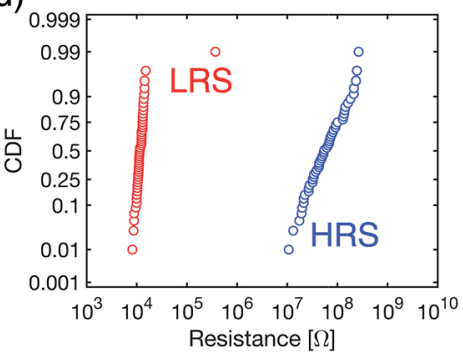

Fig. 4 Measured I-V curves for $\mathrm{HfO}_{2}$ (a) and for $\mathrm{SiO}_{x}$ (b), and the corresponding distributions of resistance for the HRS and LRS (c, d). The compliance current was $I_{C}=80 \mu \mathrm{A}$ and the stop voltage was $V_{\text {stop }}=-3.5 \mathrm{~V}$. The resistance window is about 5000 in both cases, which supports a similar mechanism for switching in the two stacks. 
positive feedback results in a self-accelerated dynamics of the CF growth at the origin of the abrupt set transition. ${ }^{13,18}$ During the set process the gate of the transistor is biased to $V_{\mathrm{G}}=1.6 \mathrm{~V}$ to limit the maximum current to $I_{\mathrm{C}}=80 \mu \mathrm{A}$. The current limitation serves as an external negative feedback mechanism to prevent irreversible breakdown of the device. Moreover, the maximum current through the device controls the size of the $\mathrm{CF},{ }^{19}$ yielding an inverse proportionality between compliance current and LRS resistance $R \propto 1 / I_{\mathrm{C}}$. The stop voltage was the same in the two sets of experiments, namely $V_{\text {stop }}=-3.5 \mathrm{~V}$.

$\mathrm{HfO}_{2}$ and $\mathrm{SiO}_{x}$ ReRAM characteristics show comparable switching behavior, indicating an average resistance window of $5 \times 10^{3}$ in $\mathrm{SiO}_{x}$ and $3 \times 10^{3}$ in $\mathrm{HfO}_{2}$. A significant difference lies in the HRS variability, which is significantly higher in $\mathrm{HfO}_{2}$. This is further confirmed by the cumulative probability distributions of the resistance values measured at $-0.5 \mathrm{~V}$ in Fig. 4 (c) and (d) for $\mathrm{HfO}_{2}$ and $\mathrm{SiO}_{x}$, respectively. The resulting relative standard deviation of the HRS is $\sigma_{\mathrm{R}} / R \approx 1$ in the $\mathrm{SiO}_{x}$ stack and $\sigma_{\mathrm{R}} / R \approx 5$ in $\mathrm{HfO}_{2}$. The larger HRS variability can be interpreted by a higher distribution of energy barriers for defect migration controlling the variation in the reset characteristics. ${ }^{8}$

The nature of resistive switching in $\mathrm{SiO}_{x}$ is attributed to the migration of $\mathrm{Ti}$ cations from the TE into the switching layer, resulting in the formation of a Ti-rich $\mathrm{CF},{ }^{10}$ and/or to the migration of oxygen vacancies leading to silicon nanoinclusions constituting the $\mathrm{CF}^{20}$ Resistive switching in $\mathrm{HfO}_{2}$ is typically attributed to oxygen vacancies, although migration of metallic cations from the active TE into the metal oxide has been postulated ${ }^{19}$ and experimentally demonstrated. ${ }^{21}$ However, the similarity of the switching characteristics in Fig. 4 in terms of shape of the $I-V$ curve and resistance window suggests that the $\mathrm{SiO}_{x}$ and $\mathrm{HfO}_{2}$ share a similar microscopic switching mechanism, which might be the migration of Ti from the TE electrode controlling the $\mathrm{CF}$ formation and disruption.

\subsection{Impact of $V_{\text {stop }}$}

During the reset process, the resistance of the ReRAM gradually increases with the applied negative voltage, as a result of the inherent negative feedback mechanism regulating the growth of the depleted gap. ${ }^{13,18}$ As a consequence, the maximum applied negative voltage $V_{\text {stop }}$ controls the length of the depleted gap and thus plays a key role to control the HRS and thus the resistance window.

The dependence of the HRS on $V_{\text {stop }}$ was studied for $t_{\mathrm{ox}}=5 \mathrm{~nm}$ and $t_{\mathrm{ox}}=10 \mathrm{~nm}$ $\mathrm{HfO}_{2}$ and for the reference $\mathrm{SiO}_{x}$ stack, and the results are reported in Fig. 5. Fig. 5 shows the measured $I-V$ curves (a) and the resistance values for the LRS and HRS as a function of $V_{\text {stop }}$ (b) for a $\mathrm{HfO}_{2}$ ReRAM with $t_{\mathrm{ox}}=5 \mathrm{~nm}$. $V_{\text {stop }}$ was changed every five set-reset cycles from $V_{\text {stop }}=-2.2 \mathrm{~V}$ to $-3.4 \mathrm{~V}$, while the compliance current was kept constant at $I_{\mathrm{C}}=50 \mu \mathrm{A}$. The results show that $V_{\text {stop }}$ controls the HRS, hence the resistance window, while the LRS remains almost unaffected by $V_{\text {stop }}$. The HRS increases exponentially at increasing $V_{\text {stop }}$ with a slope of $1.2 \mathrm{dec} \mathrm{V}^{-1}$. For $V_{\text {stop }}=-3.4 \mathrm{~V}$, the device undergoes breakdown under negative voltage, which was already shown to control endurance lifetime under pulsed set/reset cycling experiments. ${ }^{15}$ In the breakdown phenomenon, the current suddenly increases, usually resulting in a LRS with an extremely low resistance due to the $I_{\mathrm{C}}$ not being directly limited by the series FET. More results about the negative-voltage breakdown will be given in Sec. 4.1. 
(a)

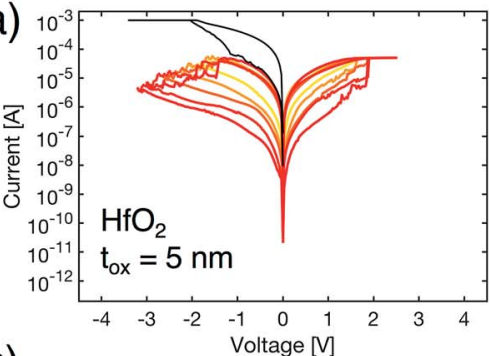

(c)

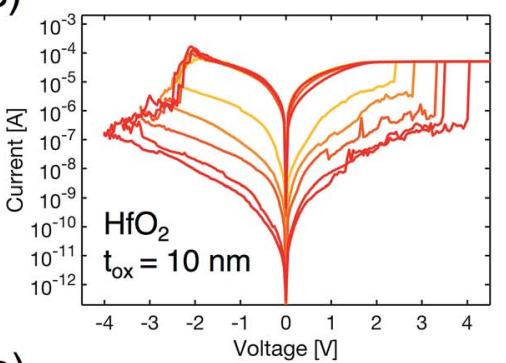

(e)

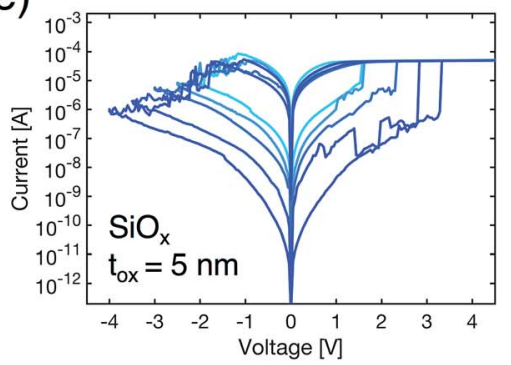

(b)

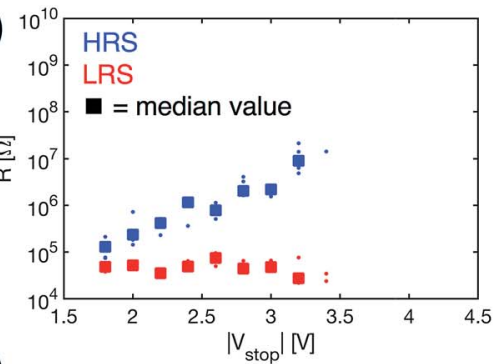

(d)

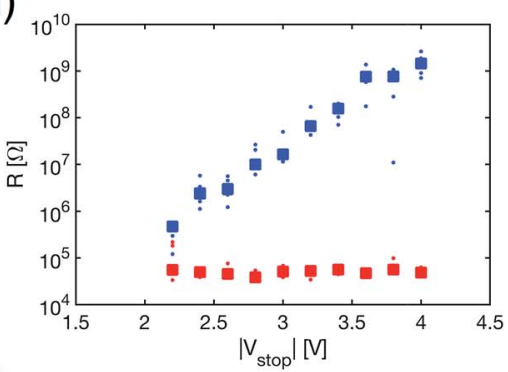

(f)

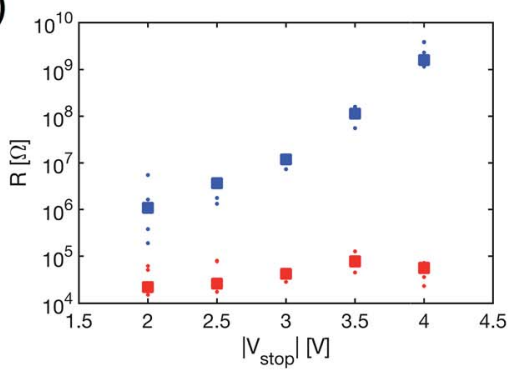

Fig. 5 Measured $I-V$ curves and resistance values as a function of $\left|V_{\text {stop }}\right|$, for $(a, b) 5 \mathrm{~nm}$ $\mathrm{HfO}_{2}$, (c, d) $10 \mathrm{~nm} \mathrm{HfO}$, and (e, f) $5 \mathrm{~nm} \mathrm{SiO}$. $\left|V_{\text {stop }}\right|$ was changed on the same device, starting from low $\left|V_{\text {stop }}\right|$ values. $I_{C}$ was kept constant at $I_{C}=50 \mu \mathrm{A}$.

Fig. 5 also shows the $I-V$ curves (c) and the resistance values (d) for the $\mathrm{HfO}_{2}$ ReRAM with $t_{\mathrm{ox}}=10 \mathrm{~nm}$. No breakdown phenomenon is seen at negative voltage, even up to $V_{\text {stop }}=-4 \mathrm{~V}$, possibly thanks to the thicker oxide layer limiting the electric field within the oxide layer. The resistance window could be effectively increased to more than $10^{4}$ at the highest $V_{\text {stop. }}$.

Data for $\mathrm{SiO}_{x}\left(t_{\mathrm{ox}}=5 \mathrm{~nm}\right)$ are also shown for comparison, including $I-V$ curves (e) and resistance values (f). The resistance values are similar to the $\mathrm{HfO}_{2}$ device with $t_{\mathrm{ox}}=10 \mathrm{~nm}$, except for the tighter control of the HRS which is promising for multilevel operation of the memory. Also notice that $V_{\text {set }}$ increases in general with the $V_{\text {stop }}$ adopted in the previous reset cycle, paralleling the increase of the HRS resistance. Excessive values of $V_{\text {set }}$ might be detrimental for ReRAM operation, which relies on low voltage operation, which suggests that a tight control of the HRS and the associated $V_{\text {set }}$ is essential in ReRAM devices.

\subsection{Switching variability}

Statistical variability of ReRAM parameters, including the HRS/LRS resistance, $V_{\text {set }}$ and $V_{\text {reset }}$, is crucial for the memory performance and the read window 
margin. To study the switching variability in the two materials, we collected cycle-to-cycle distributions of the HRS and LRS and evaluated the corresponding standard deviation $\sigma_{\mathrm{R}}$ and the average resistance $R$ for each distribution at various conditions of $I_{\mathrm{C}}$ and $V_{\text {stop }}$. Fig. 6 shows the relative standard deviation $\sigma_{\mathrm{R}} / R$ as a function of $R$ for the $\mathrm{HfO}_{2} \operatorname{ReRAM}\left(t_{\mathrm{ox}}=10 \mathrm{~nm}\right)$ and the $\mathrm{SiO}_{x}$ ReRAM $\left(t_{\mathrm{ox}}=5 \mathrm{~nm}\right) .{ }^{22}$ In the figure, LRS data were obtained at variable $I_{\mathrm{C}}$ while HRS data were obtained at variable $V_{\text {stop. }}$. The LRS variation shows similar behavior in the $\mathrm{HfO}_{2}$ and $\mathrm{SiO}_{x}$ ReRAMs, indicating a linear increase of $\sigma_{\mathrm{R}} / R$ with $R$. The latter is consistent with a variability model based on the stochastic variation of the $C F$ shape from cycle to cycle. ${ }^{23}$ A lower LRS variability was found in $\mathrm{HfO}_{2}$ for relatively large resistance values $\left(R \approx 10^{5} \Omega\right)$, namely for low $I_{\mathrm{C}}$.

HRS variation shows a slightly different behavior in the two materials, with $\mathrm{HfO}_{2}$ indicating an increase of $\sigma_{\mathrm{R}} / R$ with $R$ approximately given by $R^{0.5}$, in agreement with previous variability models based on the Poissonian statistics controlling the number of defects in the depleted gap. ${ }^{7,8}$ On the other hand, $\mathrm{SiO}_{x}$ ReRAM shows an almost constant $\sigma_{\mathrm{R}} / R \approx 1$ even for the highest $R$ values around $1 \mathrm{G} \Omega$. These data thus support the superior performance of $\mathrm{SiO}_{x}$ ReRAM in terms of cycle-to-cycle variability, which plays a key role in the ReRAM memory operation and yield.

\section{Reliability study}

\subsection{DC breakdown}

Fig. 7(a) shows $I-V$ characteristics of $\mathrm{HfO}_{2}$ ReRAM devices with $t_{\mathrm{ox}}=5 \mathrm{~nm}$ for negative voltage, indicating the typical reset process of decreasing current, and the anomalous breakdown process where the current suddenly increases at a stochastic breakdown voltage $V_{\mathrm{BD}}$. Breakdown occurs during the negative voltage sweep and leads to an LRS with extremely low resistance. Similar breakdown phenomena were observed in 1T1R structures during pulsed cycling and

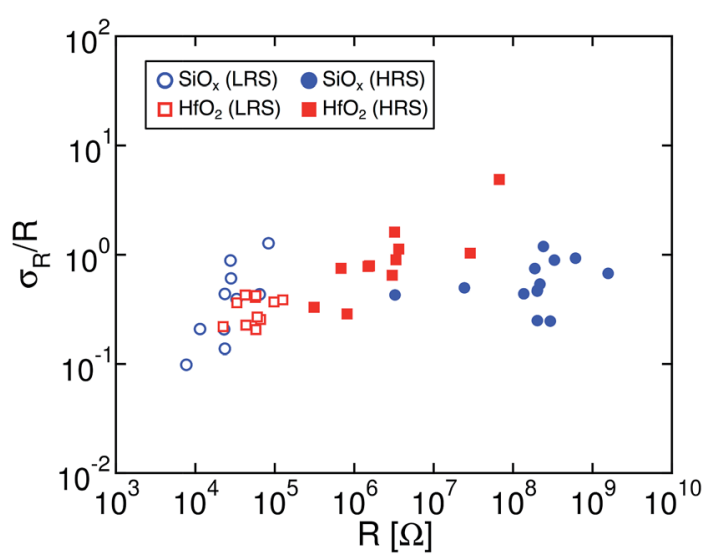

Fig. 6 Relative resistance spread $\sigma_{\mathrm{R}} / R$ as a function of the resistance $R$ for $10 \mathrm{~nm}$ thick $\mathrm{HfO}_{2}$ compared to $\mathrm{SiO}_{x}$ data in ref. 22. The data show comparable LRS variability, while the HRS is much more stable in $\mathrm{SiO}_{x}$, showing a larger window for the same relative spread, and a weaker dependence of $\sigma_{\mathrm{R}} / R$ on $R$. 
(a)

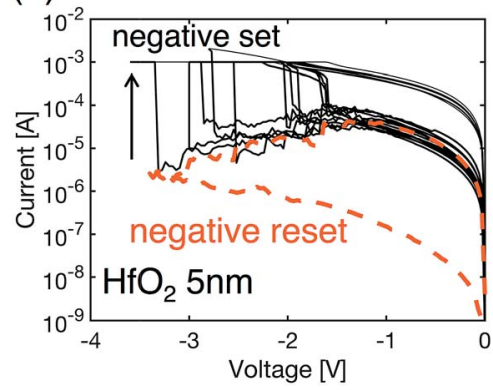

(b)

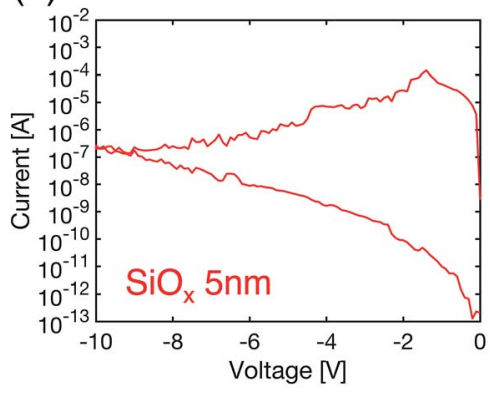

Fig. 7 (a) Typical failure mechanism in $\mathrm{HfO}_{2}$ ReRAM devices is by negative-set breakdown. (b) $\mathrm{SiO}_{x}$ devices $\left(t_{\mathrm{ox}}=5 \mathrm{~nm}\right.$ ) do not show negative-set up to very large negative voltages.

attributed to rupture of the $\mathrm{BE}$ interface and a consequent defect injection from the BE. ${ }^{15}$ Negative-set cycles are generally destructive events, causing the failure of the memory device. In fact, during the application of negative voltages the gate of the integrated series transistor is generally biased to a relatively large voltage to minimize the voltage drop across the series transistor. This results in an overgrowth of the CF during negative-set, leading to a stuck-set state. To prevent irreversible breakdown in Fig. 7(a), the current meter was set to force a compliance current of $1 \mathrm{~mA}$, thus much larger than the typical operating currents $I_{\text {reset }} \approx$ $I_{\mathrm{C}}$ of about $80 \mu \mathrm{A}$.

While $\mathrm{HfO}_{2}$ ReRAM with $t_{\mathrm{ox}}=5 \mathrm{~nm}$ systematically shows breakdown, the same is not observed in the $\mathrm{SiO}_{x}$ ReRAM with the same oxide thickness, even when relatively large negative voltages are applied, e.g., $V_{\text {stop }}=-10 \mathrm{~V}$ in Fig. $7(\mathrm{~b})$. This might be attributed to the higher energy barrier for defect migration in the $\mathrm{SiO}_{x}$ layer, compared to $\mathrm{HfO}_{2}$, which is also consistent with the higher $V_{\mathrm{FORM}}$ of $\mathrm{SiO}_{x}$ in Fig. 2.

\subsection{Retention at elevated temperature}

A key requirement for non-volatile memories is data retention at high temperatures, which might be expected in several embedded memory circuits for automotive and industrial applications. For instance, the reliability specifications of embedded ReRAM require that data remain stored after a high temperature bake annealing at $260{ }^{\circ} \mathrm{C}$ for a few minutes. ${ }^{24}$ Due to their filamentary storage, ReRAM devices can be affected by temperature-induced resistance changes, as a result of defect diffusion causing either disruption of the CF or closure of the depleted gap. ${ }^{16} \mathrm{~A}$ careful study of retention at elevated temperature is therefore crucial for validating and comparing ReRAM materials.

We studied data retention by cumulative 1 hour-annealing processes at increasing temperature $T_{\mathrm{A}}$. Multiple $\mathrm{HfO}_{2}$ and $\mathrm{SiO}_{x}$ devices with $t_{\mathrm{ox}}=5 \mathrm{~nm}$ were initially prepared in various LRS and HRS states by changing $I_{\mathrm{C}}$ and $V_{\text {stop }}$ respectively. After the initial resistance measurement, each device was annealed for 1 hour at $T_{\mathrm{A}}=120{ }^{\circ} \mathrm{C}$. The process was repeated at increasing temperature steps of $20^{\circ} \mathrm{C}$ up to a maximum annealing temperature $T_{\mathrm{A}}=260^{\circ} \mathrm{C}$. After every annealing step, resistance measurements were carried out at room temperature $T_{0}$ to avoid T-induced conductivity variations. 
(a) $\mathrm{HfO}_{2}$

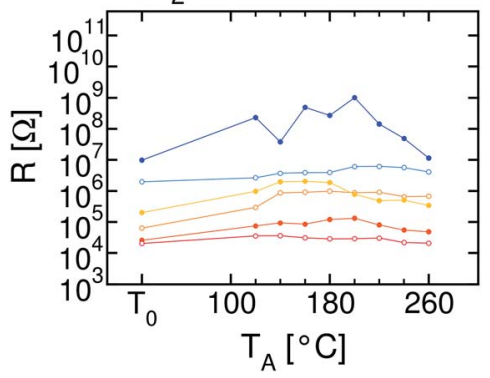

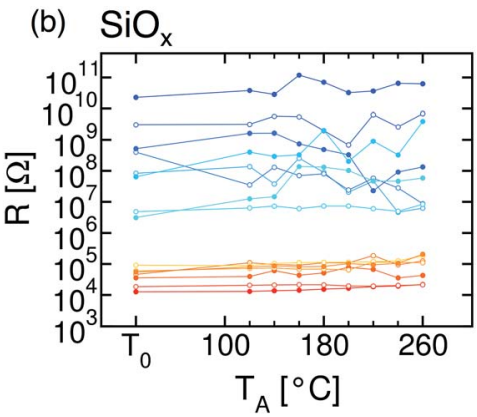

Fig. 8 Resistance measured at room temperature $T_{0}$ after cumulative $1 \mathrm{~h}$ annealing processes, as a function of the annealing temperature $T_{\mathrm{A}}$, in (a) $\mathrm{HfO}_{2}$, and (b) $\mathrm{SiO}_{x}$.

Fig. 8 shows the measured resistance as a function of the annealing temperature $T_{\mathrm{A}}$ for the $\mathrm{HfO}_{2}$ material in (a), and for the $\mathrm{SiO}_{x}$ in (b). The data show relatively good temperature stability for both materials in both the LRS and HRS. The latter shows larger resistance fluctuations, possibly explained by the redistribution of defects within the depleted gap. However, no data loss is observed, which supports the strong reliability of $\mathrm{SiO}_{x}$ ReRAM devices.

The data for $\mathrm{HfO}_{2}$ ReRAM also show a relatively small change upon temperature stress, although a smaller resistance range was explored in this case to prevent negative breakdown (see also Fig. 5(a)). Overall, the strong stability at elevated temperature observed in Fig. 8 further supports the interpretation of a common switching mechanism in $\mathrm{HfO}_{2}$ and $\mathrm{SiO}_{x}$ ReRAM, where Ti-migration is responsible for the growth and disruption of the CF. Our results thus support a strong role of the TE and BE materials in dictating the switching and reliability of ReRAM devices.

\section{Conclusions}

This paper shows a comprehensive comparison of $\mathrm{SiO}_{x^{-}}$and $\mathrm{HfO}_{2}$-based ReRAM devices, aiming at discriminating between the impact of the oxide and electrode materials in the switching and reliability performance of the device. The study is carried out for the same device geometry, electrode materials, and fabrication process, by just changing the switching layer material. The forming characteristics are considerably different in the two dielectrics, possibly highlighting a difference in energy barriers for defect migration. On the other hand, clear similarities are observed for the static $I-V$ curves; in particular, the similar resistance window suggests a central role of the TE during resistance switching. The annealing experiment evidences good stability at high temperature for both $\mathrm{HfO}_{2}$ and $\mathrm{SiO}_{x}$. Differently, switching variability characteristics are radically different in the two oxides, showing better performance for the $\mathrm{SiO}_{x}$ device.

\section{Conflicts of interest}

There are no conflicts to declare. 


\section{Acknowledgements}

This work has received funding from the European Research Council (ERC) under the European Union's Horizon 2020 research and innovation programme (Grant Agreement No. 648635). The clean room PoliFAB is acknowledged for device fabrication.

\section{Notes and references}

1 R. Waser and M. Aono, Nat. Mater., 2007, 6, 833-840.

2 H. S. P. Wong, H. Y. Lee, S. Yu, Y. S. Chen, Y. Wu, P. S. Chen, B. Lee, F. T. Chen and M. J. Tsai, Proc. IEEE, 2012, 100(6), 1951-1970.

3 Resistive Switching: From Fundamentals of Nanoionic Redox Processes to Memristive Device Applications, ed. D. Ielmini and R. Waser, John Wiley \& Sons, 2015.

4 J. J. Yang, D. B. Strukov and D. R. Stewart, Nat. Nanotechnol., 2013, 8, 13-24.

5 J. Borghetti, G. S. Snider, P. J. Kuekes, J. J. Yang, D. R. Stewart and R. S. Williams, Nature, 2010, 464, 873-876.

6 M. A. Zidan, J. P. Strachan and W. D. Lu, Nat. Electron., 2018, 1, 22-29.

7 S. Balatti, S. Ambrogio, D. C. Gilmer and D. Ielmini, IEEE Electron Device Lett., 2013, 34(7), 861-863.

8 S. Ambrogio, S. Balatti, A. Cubeta, A. Calderoni, N. Ramaswamy and D. Ielmini, IEEE Trans. Electron Devices, 2014, 61(8), 2912-2919.

9 S. Ambrogio, S. Balatti, V. McCaffrey, D. C. Wang and D. Ielmini, IEEE Trans. Electron Devices, 2015, 62(11), 3812-3819.

10 A. Bricalli, E. Ambrosi, M. Laudato, M. Maestro, R. Rodriguez and D. Ielmini, IEEE Trans. Electron Devices, 2018, 65(1), 115-121.

11 A. Bricalli, E. Ambrosi, M. Laudato, M. Maestro, R. Rodriguez and D. Ielmini, IEEE Trans. Electron Devices, 2018, 65(1), 122-128.

12 Z. Wang, S. Joshi, S. E. Savel'ev, H. Jiang, R. Midya, P. Lin, M. Hu, N. Ge, J. P. Strachan, Z. Li, Q. Wu, M. Barnell, G. L. Li, H. L. Xin, R. S. Williams, Q. Xia and J. J. Yang, Nat. Mater., 2017, 16, 101-108.

13 F. Nardi, S. Larentis, S. Balatti, D. C. Gilmer and D. Ielmini, IEEE Trans. Electron Devices, 2012, 59(9), 2461-2467.

14 A. Calderoni, S. Sills, C. Cardon, E. Faraoni and N. Ramaswamy, Microelectron. Eng., 2015, 147, 145-150.

15 S. Balatti, S. Ambrogio, Z. Wang, S. Sills, A. Calderoni, N. Ramaswamy and D. Ielmini, IEEE Trans. Electron Devices, 2015, 62(10), 3365-3372.

16 B. Traoré, P. Blaise, E. Vianello, H. Grampeix, S. Jeannot, L. Perniola, B. De Salvo and Y. Nishi, IEEE Trans. Electron Devices, 2015, 62(12), 4029-4036.

17 D. Ielmini and V. Milo, J. Comput. Electron., 2017, 16(4), 1121-1143.

18 S. Ambrogio, S. Balatti, D. C. Gilmer and D. Ielmini, IEEE Trans. Electron Devices, 2014, 61(7), 2378-2386.

19 D. Ielmini, IEEE Trans. Electron Devices, 2011, 58(12), 4309-4317.

20 A. Mehonic, S. Cueff, M. Wojdak, S. Hudziak, O. Jambois, C. Labbé, B. Garrido, R. Rizk and A. J. Kenyon, J. Appl. Phys., 2012, 111(7), 074507.

21 A. Wedig, M. Luebben, D. Y. Cho, M. Moors, K. Skaja, V. Rana, T. Hasegawa, K. K. Adepalli, B. Yildiz, R. Waser and I. Valov, Nat. Nanotechnol., 2016, 11, 67-74. 
22 A. Bricalli, E. Ambrosi, M. Laudato, M. Maestro, R. Rodriguez and D. Ielmini, 2016 IEEE International Electron Devices Meeting (IEDM), 2016, pp. 4.3.1 - 4.3.4.

23 D. Ielmini, Semicond. Sci. Technol., 2016, 31(6), 063002.

24 H. L. Lung, M. Breitwisch, J. Y. Wu, P. Y. Du, Y. Zhu, M. H. Lee, Y. H. Shih, E. K. Lai, R. Dasaka, T. Y. Wang, C. F. Chen, R. Cheek, A. Schrott, E. Joseph, H. Y. Cheng, S. Raoux and C. Lam, 2011 Symposium on VLSI Technology, 2011, pp. 98-99. 